\title{
Widening the availability of ammonia to increase the footprint of cardiac PET
}

\author{
Stephen J. Horgan, MD, PhD, ${ }^{\mathrm{a}}$ and Gary V. Heller, MD, PhD ${ }^{\mathrm{a}}$ \\ a Division of Cardiovascular Medicine, Morristown Medical Center, Gagnon Cardiovascular \\ Institute, Morristown
}

Received Nov 25, 2019; accepted Nov 25, 2019

doi: 10.1007/s12350-019-01986-4

\section{See related article, pp. 295-299}

In this issue of the Journal of Nuclear Cardiology, Pieper et al. report on their experience of an N13-ammonia compact superconducting production system for positron emission tomography myocardial perfusion imaging (PET MPI). ${ }^{1}$ Currently the production of N13ammonia, an alternate FDA approved PET tracer to rubidium-82, requires the presence of an on-site or very close proximity cyclotron. This necessity, because of the cost and complexity involved, severely limits the feasibility of this radiotracer. This new system, in which this is the first clinical report, holds promise to considerably improve availability of N13-ammonia to many more office and hospital-based practices.

Cardiac PET myocardial perfusion imaging has been gaining momentum in the cardiac imaging arena, primarily based upon its superior characteristics in comparison with other non-invasive modalities. In a recent joint Position Statement, the American Society of Nuclear Cardiology and the Society of Nuclear Medicine and Molecular Imaging noted a significant underutilization of PET MPI relative to its demonstrated advantages in the assessment of suspected or known coronary artery disease. ${ }^{2}$ The advantages of PET MPI over single photon emission computed tomography (SPECT) include high diagnostic accuracy, ${ }^{3-5}$ higher spatial and contrast resolution, correction for attenuation artifacts despite body habitus, low radiation exposure, and short acquisition times. In addition, the Position

Reprint requests: Stephen J. Horgan, MD, PhD, Division of Cardiovascular Medicine, Morristown Medical Center, Gagnon Cardiovascular Institute, Morristown; shorgan@live.ie

J Nucl Cardiol 2021;28:300-2.

1071-3581/\$34.00

Copyright (C) 2019 American Society of Nuclear Cardiology.
Statement noted the ability of cardiac PET to evaluate myocardial blood flow, which is unique in the non-invasive imaging arena. Myocardial blood flow has several attributes including substantially reducing the risk of (coronary artery disease) CAD with a normal result, determining success of vasodilator stress, providing important risk stratification and adjudicating the presence of multivessel disease. ${ }^{6}$ In this Position Statement, for the first time both organizations concluded that cardiac PET MPI was a preferred test for all patients recommended for pharmacologic stress imaging. The document also concluded that Cardiac PET MPI was strongly recommended for certain categories of patients. ${ }^{2}$ These groups include: previous inconclusive study, body habitus limiting image quality, high risk patients, previous CAD patients needing reduced radiation exposure, and in cases in which determining myocardial blood flow would be advantageous to patient care. This Statement, in addition to the surge in supportive literature, has positioned Cardiac PET as a mainstream nuclear cardiology procedure. Despite these developments, there are roughly 250 centers in the United States performing PET MPI at present which is a small fraction of the number of SPECT cameras throughout the country (estimated to be approximately 5000). The vast majority of these centers are using rubidium- 82 with circa 20 centers nationwide, mostly at university teaching hospitals, are engaging in N13-ammonia programs. Both agents are ideally suited to pharmacologic stress, hence the societal recommendations for "preferred" status for this form of stress alone.

Currently, rubidium-82 and N13-ammonia are the only two FDA approved radiopharmaceuticals for PET MPI. The novel radiopharmaceutical $18 \mathrm{~F}$-flurpiridaz is currently in phase 3 clinical trials. The Phase 2 and 3 Trial data were encouraging and the final Phase 3 Trial is nearing completion. Until that time, Rubidium- 82 remains the most widely used PET tracer throughout the United States and Europe due to the distinct advantage of not requiring an 
on-site cyclotron but a generator which can be delivered on-site. The stable availability of strontium has provided significant growth in the number of centers performing cardiac PET MPI from 40 generators delivered 15 years ago to over 200 annually today. The literature quoted by the authors of the ASNC/SNMMI Position Statement, although containing data from both rubidium-82 and N13ammonia, is primarily that of rubidium perhaps due to its greater use in laboratory settings.

Despite this success, rubidium-82 has several potential limitations in relation to N13-ammonia. Importantly, the spatial resolution of N13-ammonia is excellent due to its shorter positron range, resulting in higher quality images (Figures 1$)^{7}$ In addition, the firstpass cardiac extraction fraction of N13-ammonia is higher $(\sim 80 \%)$ compared to rubidium $(\sim 65 \%)$ which potentially provides more accurate and reproducible blood flow measurement. ${ }^{7}$ Lastly, rubidium is limited to pharmacological stress testing due to its very short halflife of 76 seconds. In contrast, N13-ammonia has a halflife of 10 minutes which provides an opportunity for use in an exercise protocol (albeit sacrificing coronary flow reserve). In terms of N13-ammonia, there are some limitations as well. Apart from production, a downside to N13-ammonia compared to rubidium is the longer study protocol which is roughly 70 minutes compared to 30 minutes. ${ }^{8}$ However, this is still considerably shorter in comparison to a standard SPECT MPI, ${ }^{9}$ and new approaches such as a lower dose rest study and higher dose stress study may reduce the overall protocol length. In addition, clinicians have noted a lateral wall defect with ammonia that is artifactual and unexplained. ${ }^{10}$ In total, N13-ammonia provides an important alternative to rubidium- 82 with both strengths and weaknesses. Having two available tracers in the PET environment offers more opportunities for users.
The major limitation for the use of N13-ammonia is the requirement of a nearby cyclotron. A cyclotron is a particle accelerator whereby charged particles accelerate outwards from a central source along a spiral path as opposed to linear particle accelerators. Their primary use in nuclear medicine is for the production of radionuclides. In addition to nearby proximity, generally there is also the need for a large space to house an onsite cyclotron, staff to support and operate the equipment as well as the high installation and running costs. For these reasons, there has been a drive to develop a more feasible compact production system. Advances in superconducting cyclotron design and developments in the automated multi-use purification unit have resulted in the evolution of a minimally shielded mini-cyclotron system. This unit can be housed in a much smaller footprint with fewer personnel to make it more costeffective and deliver unit doses to communities not currently able to consider N13-ammonia.

In their paper published in this issue of the Journal of Nuclear Cardiology, Pieper and associates describe their initial clinical experience with a mini-cyclotron system (ION-12SC system-Ionetix, Inc., San Francisco, CA). Ninety-seven patients underwent imaging with N13 ammonia over the course of 4 weeks from the minicyclotron system. All patients were injected with $0.4 \mathrm{mg}$ of regadenoson intravenously but the study did not include any patients undergoing an exercise protocol. Roughly two-thirds of the patients had a rest/stress protocol with the remainder having either rest or stress only studies. Overall scan quality was graded based on image noise, contrast, patient motion and quality of tracer bolus administration. The vast majority of studies were considered to have good or excellent image quality $(91.8 \%)$ as interpreted by three board certified nuclear cardiologists. The median length of time for a rest-stress
${ }^{15} \mathrm{O}$ water

Positron range $(\mathrm{mm})$

4.14

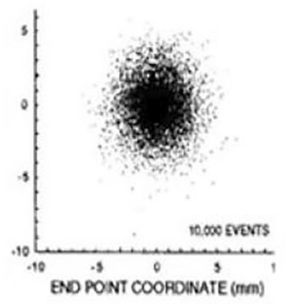

${ }^{13} \mathrm{~N}$ ammonia

2.53

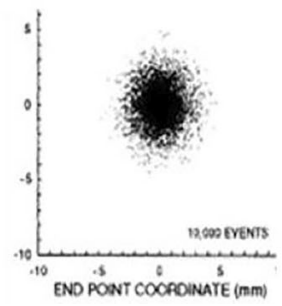

8.6

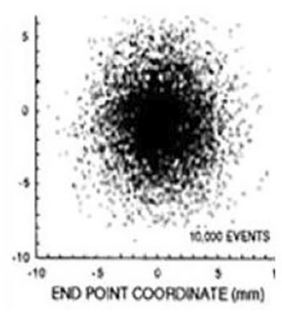

${ }^{18} \mathrm{~F}$ flurpiridaz

1.03

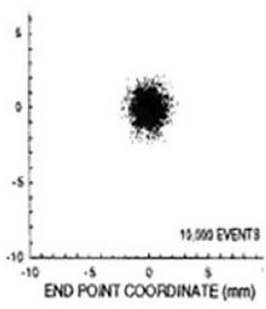

Figure 1. Positron range of perfusion PET tracers. 
protocol was 48.1 minutes. The tracer related radiation exposure was low at 2.1 to $4 \pm 0.1 \mathrm{mSv}$ for a rest-stress protocol and very low for a rest only study at $0.7 \pm 0.1 \mathrm{mSv}$. There were no major complications and no adverse reactions were observed.

The present brief report is limited by a small number of patients, the lack of comparison with N13-ammonia PET imaging from a traditional cyclotron, and a lack of any data on coronary blood flow. However, it is a welcome proof of concept observational study with promising results and represents the first study assessing the clinical performance of an automated N13-ammonia mini cyclotron. In the era of high value initiatives, regardless of body habitus, myocardial perfusion PET is preferred and recommended in patients requiring pharmacological nuclear stress testing (and select cases for exercise with N13ammonia). The compact superconducting production system for PET MPI (mini cyclotron) promises to expand the utilization of this superior radiopharmaceutical through improved access and deliverability. Cost will remain a significant factor and a mobile mini cyclotron may alleviate some of these expenditure issues permitting wider use of N13-ammonia. How this concept of a mini cyclotron in clinical practice will actually work from a logistic and monetary point of view remains to be seen. However, the cardiac PET community should welcome this development as an opportunity to further expand this very important and clinically useful imaging tool in an era of a plethora of multimodality imaging options.

Positron range and end point coordinates are shown for various myocardial perfusion PET tracers. The higher the energy of the emitted positron, the longer it travels away from the source before annihilation and the worse the resolution of the imaged target. In this figure, end point coordinates are similar to point source images obtained from a given PET tracer (reprinted from Seminars in Nuclear Medicine volume 44 edition 5; Maddahi J, Packard RR; Cardiac PET perfusion tracers: current status and future directions; pages 333-43, 2014 with permission from Elsevier). ${ }^{7}$

\section{Disclosures}

Gary V. Heller was supported by PMA Medical GE Healthcare MIS and received royalties form McGraw-Hill Publishing. Stephen J. Horgan declares that he has no financial conflict of interest.

\section{References}

1. Pieper J, Patel VN, Escolero S, Nelson JR, Poitrasson-Riviere A, Shreves CK, et al. Initial clinical experience of N13-ammonia myocardial perfusion PET/CT using a compact superconducting production system. J Nucl Cardiol 2019. https://doi.org/10.1007/ s12350-019-01886-7.

2. Bateman TM, Dilsizian V, Beanlands RS, DePuey EG, Heller GV, Wolinsky DA. American Society of Nuclear Cardiology and Society of Nuclear Medicine and Molecular Imaging Joint Position Statement on the clinical indications for myocardial perfusion PET. J Nucl Med 2016;57:1654-6.

3. Nandalur KR, Dwamena BA, Choudhri AF, Nandalur SR, Reddy P, Carlos RC. Diagnostic performance of positron emission tomography in the detection of coronary artery disease: a metaanalysis. Acad Radiol 2008;15:444-51.

4. Parker MW, Iskandar A, Limone B, Perugini A, Kim H, Jones C, et al. Diagnostic accuracy of cardiac positron emission tomography versus single photon emission computed tomography for coronary artery disease: a bivariate meta-analysis. Circ Cardiovasc Imaging 2012;5:700-7.

5. Mc Ardle BA, Dowsley TF, deKemp RA, Wells GA, Beanlands RS. Does rubidium-82 PET have superior accuracy to SPECT perfusion imaging for the diagnosis of obstructive coronary disease?: A systematic review and meta-analysis. J Am Coll Cardiol 2012;60:1828-37.

6. Murthy VL, Bateman TM, Beanlands R, et al. Clinical quantification of myocardial blood flow using PET: Joint position paper of the SNMMI cardiovascular council and the ASNC. J Nucl Cardiol. 2018;25:269-97.

7. Maddahi J, Packard RR. Cardiac PET perfusion tracers: Current status and future directions. Semin Nucl Med 2014;44:333-43.

8. Dilsizian V, Bacharach SL, Beanlands RS, Bergmann SR, Delbeke $\mathrm{D}$, Dorbala S, et al. ASNC imaging guidelines/SNMMI procedure standard for positron emission tomography (PET) nuclear cardiology procedures. J Nucl Cardiol 2016;23:1187-226.

9. Henzlova MJ, Duvall ML, Einsteir AJ, et al. ASNC imaging guidelines for SPECT nuclear cardiology procedures: Stress, protocols, and tracers. J Nucl Cardiol 2016;21:1071.

10. Di Carli MF, Murthy VL. Cardiac PET/CT for the evaluation of known or suspected coronary artery disease. Radiographics 2011;31:1239-54.

Publisher's Note Springer Nature remains neutral with regard to jurisdictional claims in published maps and institutional affiliations. 